\section{Antipsychotics and diabetes: review of non-prospective data}

PETER M. HADDAD
An association between antipsychotic medication and diabetes mellitus and/or impaired glucose tolerance was reported in the 1950s, soon after chlorpromazine entered clinical practice (Hiles, 1956). Other reports followed (e.g. Cooperberg $\&$ Eidlow, 1956). Although many of these reports had methodological shortcomings, by the late 1960 s there was a general acceptance that antipsychotic drugs could cause diabetes, and the term 'phenothiazine diabetes' was coined (ThonnardNeumann, 1968). There was little further interest in the subject until recently, when attention became focused on the relationship between the atypical antipsychotics, and impaired glucose tolerance and diabetes. In late 2003 the Food and Drug Administration (FDA) recommended updated product labelling for all atypical antipsychotics licensed in the USA, to include a warning about their association with hyperglycaemia and diabetes.

Evidence for an association between antipsychotics and diabetes can be gleaned from many sources, including prevalence studies, anecdotal reports, retrospective case-note studies, pharmaco-epidemiological studies, 'physiological' studies assessing various parameters relevant to control of blood glucose, and prospective studies. Prospective studies represent the highest level of evidence and should carry the most weight. Nevertheless, it is important to evaluate all sources of data when considering such an important issue. This paper therefore provides a critical review of the first four data sources listed above. Before considering these data, it is important to review risk factors for diabetes.

\section{Risk factors for diabetes}

\section{Socio-demographic and lifestyle factors}

Any study that attempts to determine whether there is a causal relationship between antipsychotic medications and diabetes needs to account for the multifactorial aetiology of the disorder. Risk factors for diabetes are:

(a) family history of diabetes mellitus

(b) increasing age

(c) obesity

(d) race (a higher incidence is reported in people of Asian and African-Caribbean origin)

(e) smoking

(f) diet (high in carbohydrates)

(g) lack of exercise

(h) diabetogenic drugs (e.g. thiazide diuretics, corticosteroids)

(i) schizophrenia?

A recurrent problem with using nonprospective data is that, by their very nature, such confounders are often ignored.

Diabetes mellitus has a high prevalence rate in most Western countries, and this has increased dramatically in the past decade. In the UK, it has been estimated that approximately 1.4 million people have been diagnosed with diabetes and that a further 1 million people have the disorder but are undiagnosed (Bennett et al, 1995). Consequently, if diabetes is diagnosed for the first time during antipsychotic treatment, a causal relationship cannot automatically be assumed; the diabetes might have existed prior to initiation of antipsychotic treatment, but without being identified.

\section{Schizophrenia}

Several studies from the pre-antipsychotic era reported insulin resistance and abnormalities of glucose homoeostasis in patients with schizophrenia (e.g. Lorenz, 1922; Braceland et al, 1946; Freeman, 1946). This can be explained either in terms of an intrinsic association between schizophrenia and impaired glucose tolerance, or as a result of aspects of lifestyle that are risk factors for impaired glucose tolerance being more common in patients with schizophrenia than in the general population. For example, patients with schizophrenia living in the community have been shown to exercise less, smoke more and have less healthy diets than a control group (Brown et al, 1999).

Ryan et al (2003) studied drug-naïve patients with first-episode schizophrenia, and so avoided the potential confounding effect of antipsychotic medication. Of 26 patients with first-episode schizophrenia, 4 $(15 \%)$ had impaired fasting glucose tolerance, compared with none of a control 
group of healthy individuals matched for age, gender and various lifestyle and anthropometric measures. The patients had significantly higher fasting plasma levels of glucose and insulin than the control group. Although this study is consistent with reports from the pre-neuroleptic era, and suggests an independent association between diabetes and schizophrenia, it is limited by the small number of participants enrolled.

Studies of patients with newly diagnosed schizophrenia who had not yet commenced antipsychotic treatment have suggested there is also a higher prevalence of metabolic syndrome than in age-matched controls (Ryan \& Thakore, 2002; Thakore et al, 2002). Several studies have reported that patients with schizophrenia are more likely to have a family history of diabetes than the general population, suggesting that there may be a genetic association between schizophrenia and diabetes (e.g. Mukherjee et al, 1989).

If schizophrenia is an independent risk factor for diabetes mellitus, and at present this can only be regarded as a hypothesis, then it is possible that the risk conferred may vary depending on the subtype of the illness, its duration and severity.

\section{METHOD}

A Medline search (1966-2003) was undertaken, supplemented by manual searches of journals and conference abstracts, to identify non-prospective studies.

\section{RESULTS}

\section{Prevalence studies}

Several studies have used a cross-sectional design to determine the prevalence of diabetes, and in some cases impaired glucose tolerance, in patients with schizophrenia. When interpreting the results, one needs to make comparisons with local populations that are matched for age; the prevalence of diabetes in the general population increases markedly with age and varies between countries. A consistent finding across these studies is that the rates of diabetes and impaired glucose tolerance are higher than in the comparative population.

Prior to the introduction of the atypical antipsychotics, a study in Japan assessed
248 patients and reported a prevalence of type 2 diabetes of $8.8 \%$, compared with $5 \%$ in a control group matched for age and gender (Tabata et al, 1987). In a US study (Dixon et al, 2000), conducted in 1994-1996, self-reported rates of diabetes in patients with schizophrenia were $14.9 \%$ (lifetime) and $10.8 \%$ (current); in contrast, the self-reported rate of diabetes in the general population of the USA in 1994 was $1.2 \%$ for people aged 18-44 years and $6.3 \%$ for people aged 45-64 (Adams \& Marano, 1995). In Italy, Mukherjee et al (1996) reported the prevalence of diabetes - assessed by fasting blood glucose levels - as $15.8 \%$. The expected prevalence in the general Italian population was $2.1-3.2 \%$.

Most recently, Subramaniam et al (2003) assessed patients with schizophrenia on long-stay psychiatric wards in Singapore. Patients were excluded if they were known to have diabetes $(4.9 \%$ of the original sample), had drug or alcohol problems, or had significant physical illness. The 194 patients who entered the study were given an oral glucose tolerance test after an overnight fast. World Health Organization (WHO) diagnostic criteria for the test were used to diagnose diabetes and impaired glucose tolerance. The overall prevalence of diabetes mellitus was $16.0 \%$ and that of impaired glucose tolerance was $30.9 \%$, higher than comparative rates in the general population $(9 \%$ and $15 \%$ respectively according to figures quoted by Subramaniam et al, 2003). Polypharmacy prevented these researchers from investigating whether rates differed in patients prescribed different antipsychotics. However, none of the patients in this study had received an atypical antipsychotic, either in the past or at the time of the study. A study in London has also shown that most cases of diabetes and impaired glucose tolerance in inpatients with schizophrenia are undiagnosed and are only revealed by blood glucose screening (Taylor et al, 2003).

In summary, cross-sectional studies demonstrate that diabetes and impaired glucose tolerance are more prevalent in patients with schizophrenia than in the general population. It is also apparent that much of this morbidity is undiagnosed and is only revealed with systematic screening. Cross-sectional studies can only demonstrate associations, they cannot determine whether antipsychotic medication is a risk factor for diabetes or impaired glucose tolerance.

\section{Anecdotal reports}

Anecdotal data regarding antipsychotic drugs and diabetes are available from spontaneous adverse drug reaction reports, i.e. the 'yellow card' system in the UK and its equivalent in other countries. However, only a small fraction of adverse drug reactions are reported in this way, and these reports cannot be used to determine the incidence of an adverse reaction or to compare rates between drugs.

Published case reports are another source of information, but they contain a further element of bias as they depend on an individual clinician's interest in submitting a report, and on editorial selection policies. Despite their weaknesses, anecdotal reports are important, as they can be the first indication of an adverse effect that was not identified in clinical trials.

\section{Conventional antipsychotics}

In 1956, Hiles described five patients who developed hyperglycaemia and glycosuria soon after starting chlorpromazine, with resolution on stopping the drug (Hiles, 1956). A further five patients with well-controlled diabetes were also described, in whom diabetic control deteriorated after they were prescribed chlorpromazine (Hiles, 1956); in all five, diabetic control returned to its pre-treatment level when chlorpromazine was withdrawn. Other reports of similar reactions to chlorpromazine followed. For example, Cooperberg \& Eidlow (1956) reported the case of a man with schizophrenia who developed diabetes 12 days after starting chlorpromazine. ThonnardNeumann (1968) reported that the prevalence of diabetes in psychiatric in-patients had increased from $4.2 \%$ in 1954 to $17.2 \%$ in 1966 , and he attributed this to the introduction of phenothiazines in the intervening period.

\section{Atypical antipsychotics}

Koller and colleagues have published several analyses of anecdotal reports of diabetes occurring with clozapine (Koller et al, 2001), olanzapine (Koller \& Doraiswamy, 2002), risperidone (Koller et al, 2003) and quetiapine (E. Koller, personal communication, 2003). All their research has been based on an analysis of published case reports plus reports made to the FDA MedWatch surveillance programme. In each paper, newly diagnosed diabetes was defined as a fasting glucose level of 
$126 \mathrm{mg} / \mathrm{dl}(7 \mathrm{mmol} / \mathrm{l})$ or greater, a random glucose level of $200 \mathrm{mg} / \mathrm{dl}(11 \mathrm{mmol} / \mathrm{l})$ or greater, or elevated glycohaemoglobin values; and/or presence of metabolic acidosis or ketosis, and/or physician initiation of an antidiabetic drug.

The Koller data are summarised in Table 1. For each of the four drugs assessed, the majority of reports occurred within 6 months of the drug's first prescription and there was a spectrum of reported abnormalities, including hyperglycaemia, diabetes, ketoacidosis and hyperosmolar coma. For each drug, a number of fatalities were reported in association with hyperglycaemia.

Hedenmalm et al (2002) published an analysis of all reports suggestive of glucose intolerance associated with risperidone, clozapine and olanzapine, identified in the WHO database for adverse drug reactions. The analysis comprised reports of the following diagnoses: glucose tolerance abnormal, hyperglycaemia, diabetes mellitus, diabetes mellitus aggravated, ketosis, diabetic coma and glycosuria. Although pharmacovigilance studies cannot provide any quantitative comparison of risk, they can provide a qualitative description of the range of glucose abnormalities reported. In the WHO data, each of the antipsychotics assessed was associated with the full range of diagnoses (Fig. 1).

Limitations of both the Koller and WHO data include variation between reports in terms of the recording of risk factors for diabetes, and the fact that most reports had no good means of assessing causality. Furthermore, a reporting bias cannot be excluded. Nevertheless, in both data-sets the relatively young age of the patients and the detection of most cases of

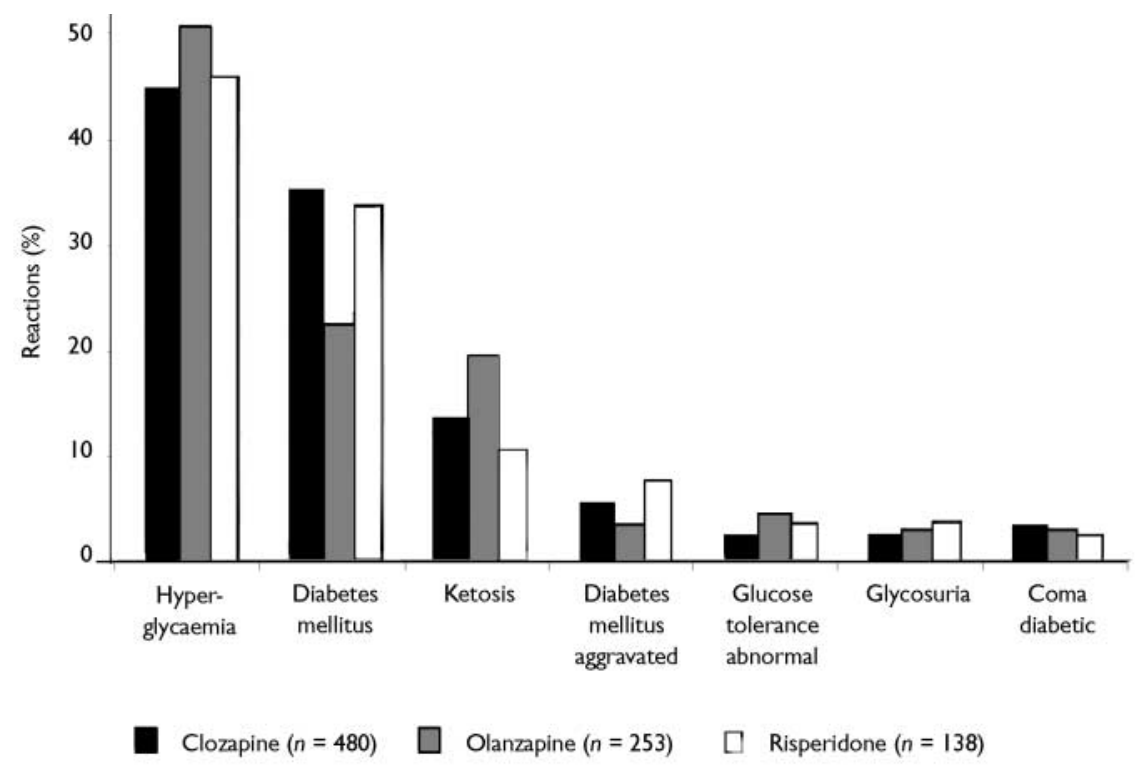

Fig. I Types of glucose abnormalities reported in patients taking atypical antipsychotic agents. Adapted with permission from Hedenmalm et al (2002), Drug Safety, 25, II07-III6.

diabetes (where data on the temporal relationship were available) within 6 months of starting the antipsychotic medication suggest a causal relationship. In addition, within both data-sets and for each of the antipsychotic medications investigated, there was a subset of patients in whom the dosage of the antipsychotic was reduced or an alternative antipsychotic was prescribed, and in a high proportion of these patients, diabetic control improved or the diabetes resolved. A small group of patients were then challenged with the original antipsychotic, and in the majority the diabetes reappeared. Both patient subsets provide further evidence that diabetes may be caused by antipsychotic medications.
The literature review conducted for this paper did not identify any published case report of diabetes associated with amisulpride. This may reflect the fact that amisulpride is not licensed in the USA; alternatively, it may reflect a lower propensity for amisulpride to cause diabetes compared with other atypical antipsychotics. It is interesting to note that among the atypical antipsychotics, amisulpride has a distinct pharmacological profile, being a pure dopamine $\mathrm{D}_{2}$ and $\mathrm{D}_{3}$ receptor antagonist. However, its diabetogenic potential remains to be confirmed - ideally in comparative prospective studies. Certainly, adverse drug reaction reports of diabetes associated with amisulpride, and with

Table I Summary of anecdotal reports of diabetes mellitus in patients taking atypical antipsychotic medications

\begin{tabular}{|c|c|c|c|c|c|c|c|}
\hline Drug & Period & $\begin{array}{c}\text { Total } \\
\text { cases' }^{\prime} \\
(n)\end{array}$ & $\begin{array}{c}\text { New-onset } \\
\text { cases } \\
\text { (n) }\end{array}$ & $\begin{array}{c}\text { Exacerbations } \\
\text { of pre-existing } \\
\text { disease }(n)\end{array}$ & $\begin{array}{c}\text { Mean } \\
\text { patient } \\
\text { age (years) }\end{array}$ & $M: F$ & $\begin{array}{l}\text { Death } \\
\text { (n) }\end{array}$ \\
\hline Clozapine (Koller et al, 200 I) & Jan 1990-Feb $200 \mathrm{I}$ & 384 & 242 & 54 & 40 & 2.0 & 25 \\
\hline Olanzapine (Koller \& Doraiswamy, 2002) ${ }^{2}$ & Jan 1994-Feb 2002 & 289 & 225 & 57 & 43 & 1.8 & 23 \\
\hline Risperidone (Koller et al, 2003) & Jan 1993-Feb 2002 & $|3|$ & 78 & 46 & 40 & 1.5 & 4 \\
\hline $\begin{array}{l}\text { Quetiapine (E. Koller, personal } \\
\text { communication, 2003) }\end{array}$ & Jan 1997-Aug 2002 & 46 & 34 & 8 & 35 & 1.9 & II \\
\hline
\end{tabular}

M:F, male/female ratio.

I.The total number of cases exceeds the sum of the new-onset cases and the exacerbations because some cases could not be categorised.

2. Includes data provided in an addendum to the main paper.

3. For an enlarged data-set see Koller et al (2004). 
the related compound sulpride, have been sent to the Committee on Safety of Medicines in the UK (CSM, personal communication, 2003).

In summary, anecdotal reports indicate that conventional and atypical antipsychotics can cause or worsen pre-existing diabetes mellitus in some patients. However, anecdotal reports cannot determine how often this occurs, or whether there is a differential risk between antipsychotic agents.

\section{Case-note studies}

Several retrospective case-note studies have investigated the relationship between atypical antipsychotics and diabetes. Meyer (2002) reviewed the charts of patients at Oregon State Hospital who were receiving treatment with either risperidone or olanzapine. To be eligible for inclusion in the study, patients had to be at least 18 years old, have had a baseline weight measurement within 3 weeks of initiation of the drug, a baseline fasting blood glucose measurement within 3 months of initiation of the drug and a fasting blood glucose measurement after 1 year of treatment ( \pm 4 weeks). Patients who had received more than 4 weeks' treatment with a second antipsychotic agent during the first year of treatment with olanzapine or risperidone were excluded. From an initial sample of 175 olanzapine-treated patients and 155 risperidone-treated patients, 47 patients from each group fulfilled the inclusion criteria.

At baseline, there was no significant difference between the risperidone- and olanzapine-treated patients in terms of body mass index (BMI), age or fasting blood glucose concentration. Over the 1year assessment period, the mean blood glucose concentration increased significantly within the olanzapine cohort $(+7.26 \mathrm{mg} / \mathrm{dl})$, whereas there was a nonsignificant increase in the risperidone cohort $(+0.68 \mathrm{mg} / \mathrm{dl})$. One of the 94 patients developed diabetes during the study; this patient had been treated with olanzapine. Although the study shows that olanzapine was associated with a significantly greater increase in blood glucose concentrations than was risperidone, the mean blood glucose levels did not differ significantly between the two drug groups at follow-up.

This study has several weaknesses. Many patients were excluded because their notes contained insufficient data, suggesting that the study might not have included a representative sample population. The final sample population in the study was predominantly White and male. Also, it is unclear in this study whether the two cohorts differed in terms of risk factors for diabetes (e.g. exercise level, diet and family history of diabetes).

Wirshing et al (2002) conducted a study with a similar design. In this study, patient notes were reviewed for 2.5 years before and after starting treatment with a range of antipsychotic medications. A significant increase in glucose levels was reported with olanzapine, clozapine and haloperidol, but not with quetiapine, risperidone or fluphenazine. However, a large proportion of the initial patient sample was excluded because the case notes did not contain enough data to allow analysis, raising questions about how representative the final sample was. Another weakness of this study was that it was unclear whether the blood glucose levels recorded in the notes were fasting samples or not.

Yu et al (2002) undertook a retrospective review of the psychiatric notes of 22 patients who had received a diagnosis of diabetes mellitus before beginning treatment with olanzapine, risperidone or quetiapine. The authors reported that no antipsychotic medication was associated with a worsening of blood glucose levels, although some treated with quetiapine or risperidone required an increase in their dose of antidiabetic medication during the study. Four of the 11 olanzapine-treated patients showed a reduction in fasting blood glucose levels and a favourable modification in their antidiabetic regimens during the study. This study also has several weaknesses, including the small sample size and the short duration of treatment - the mean duration of antipsychotic treatment was only 36.6 days. Most importantly, no information is given about the degree of diabetic control prior to the study.

In summary, two retrospective casenote studies (Meyer, 2002; Wirshing et al, 2002) have shown that several antipsychotic medications (olanzapine, clozapine and haloperidol) are associated with an increase in mean blood glucose levels during treatment lasting 1 year or more. These studies cannot determine whether the increase was caused by the antipsychotic medication or by other factors. The lack of a significant increase in mean blood glucose concentration in one or more comparator groups treated with other antipsychotic medications suggests a causal effect. However, this must remain speculative, given that neither study adequately controlled for risk factors for diabetes, and that the final samples might not have been representative of the larger population of people with schizophrenia.

\section{Pharmaco-epidemiological studies}

A number of pharmaco-epidemiological studies investigating the association between diabetes mellitus and antipsychotic medications have recently been published or presented at conferences. Most of these studies have been sponsored by the pharmaceutical industry, and all rely on the analysis of computerised databases of patients treated in various organisations, and are limited by the quality of clinical data recorded. These studies compare the risk of diabetes in cohorts of patients treated with one or more atypical antipsychotics with the risk in patients not taking antipsychotics and/or using conventional antipsychotics. Pharmaco-epidemiological studies can be cross-sectional in design and determine prevalence rates of diabetes (e.g. Sernyak et al, 2002), but most assess the risk of new cases of diabetes appearing during a specified observation period - i.e. they assess incidence rates. Before considering the results of these studies, it is important to highlight their strengths and weaknesses.

\section{Methodological issues}

Pharmaco-epidemiological studies include large samples of 'real patients' who may be more susceptible to developing diabetes than the carefully selected patients who enter clinical trials. However, the pharmacoepidemiological approach has four main weaknesses:

(a) lack of standardisation with regard to the detection and diagnosis of diabetes;

(b) failure to account for risk factors for diabetes;

(c) failure to consider all atypical antipsychotics;

(d) failure to account for variation in aspects of the patients' antipsychotic treatment and underlying psychiatric illness.

Pharmaco-epidemiological studies rely on a clinical diagnosis of diabetes. However, there is variation between studies as to how diabetes is diagnosed, and the level of screening undertaken. For example, Buse 
et al (2003) used the prescription of antidiabetic medication as a proxy for the diagnosis, thereby excluding all patients with the condition whose treatment involved dietary and lifestyle changes only. In addition, without systematic screening of blood glucose levels in all patients, many cases of diabetes are likely to be missed, and the results could be distorted through differential detection as a result of variations in screening (Citrome, 2003).

The second weakness of the pharmacoepidemiological approach is that many such studies fail to account for risk factors associated with diabetes such as diet, exercise level, smoking status, race, family history of the disorder, and BMI. In most of these studies it has been impossible to control for these confounders, as the information required is not available within the database.

The third weakness is that pharmacoepidemiological data are not available for all the atypical antipsychotic agents. Most of these studies have been conducted in the USA, and so exclude amisulpride and zotepine because these drugs are not licensed there. Several studies have excluded quetiapine and ziprasidone owing to the small numbers of patients receiving these treatments at the time the data were collected.

Finally, these studies do not control for key aspects of the patients' psychiatric illness and antipsychotic treatment. For example, Koro et al (2002) assessed patients over a 3-month period, but it is unclear how long patients had been receiving the specified class of antipsychotic prior to this. As already discussed, there is evidence that schizophrenia may be an independent risk factor for diabetes, yet none of the studies reviewed controlled for length or severity of schizophrenia. Indeed, some studies were not restricted to patients with schizophrenia, but included all patients prescribed antipsychotic medications, irrespective of psychiatric diagnosis.

\section{Pharmaco-epidemiological study results}

Tables 2 and 3 summarise the results of the pharmaco-epidemiological studies published or presented at conferences to date. Although the results are conflicting, two themes emerge. Most studies report a higher risk of diabetes developing in patients treated with antipsychotics compared with those who are not (Table 2; Koro et al, 2002; Gianfrancesco et al, 2002; Cavazzoni et al, 2002; Buse et al, 2003), and in

Table 2 Summary of pharmaco-epidemiological studies assessing risk of developing diabetes in patients taking antipsychotics $v$. patients not using these drugs

\begin{tabular}{|c|c|c|c|}
\hline Study & Database & Funding' & Risk of diabetes \\
\hline Koro et al (2002) & UK GP database & BMS & $\begin{array}{l}\text { Increased with olanzapine and conventional } \\
\text { antipsychotics } \\
\text { No increase with risperidone }\end{array}$ \\
\hline $\begin{array}{l}\text { Gianfrancesco et al } \\
(2002)\end{array}$ & $\begin{array}{l}\text { US health plan } \\
\text { database }\end{array}$ & Janssen & $\begin{array}{l}\text { Increased with clozapine, olanzapine, } \\
\text { low-potency conventionals, high-potency } \\
\text { conventionals } \\
\text { No increase with risperidone }\end{array}$ \\
\hline $\begin{array}{l}\text { Cavazzoni et al } \\
(2002)\end{array}$ & UK GP database & Lilly & $\begin{array}{l}\text { Increased with risperidone and thioridazine } \\
\text { No analysis for other agents }\end{array}$ \\
\hline Buse et al (2003) & $\begin{array}{l}\text { US prescription } \\
\text { claims database }\end{array}$ & Lilly & $\begin{array}{l}\text { Increased with clozapine, olanzapine, } \\
\text { quetiapine, risperidone, haloperidol, } \\
\text { thioridazine, combined conventionals, } \\
\text { combined atypicals }\end{array}$ \\
\hline
\end{tabular}

GP, general practitioner.

I. BMS, Bristol-Myers Squibb; Janssen, Janssen-Cilag; Lilly, Eli Lilly \& Co.

Table 3 Summary of pharmaco-epidemiological studies assessing risk of developing diabetes in patients taking atypical antipsychotics $v$. patients taking conventional antipsychotics

\begin{tabular}{|c|c|c|c|}
\hline Study & Database & Funding' & Risk of diabetes \\
\hline Sernyak et al (2002) & Veterans database & Independent & $\begin{array}{l}\text { Increased with clozapine, quetiapine, } \\
\text { olanzapine } \\
\text { No increase with risperidone }\end{array}$ \\
\hline Koro et al (2002) & UK GP database & BMS & $\begin{array}{l}\text { Increased with olanzapine } \\
\text { No increase with risperidone }\end{array}$ \\
\hline Lambert et al (2002) & Medicaid claims & BMS & $\begin{array}{l}\text { Increased with clozapine, quetiapine, } \\
\text { olanzapine } \\
\text { No increase with risperidone }\end{array}$ \\
\hline Fuller et al (2003) & Veterans database & Janssen & Olanzapine $>$ risperidone \\
\hline Buse et al (2003) & $\begin{array}{l}\text { US prescription } \\
\text { claims database }\end{array}$ & Lilly & $\begin{array}{l}\text { No increase with combined atypical cohort } \\
\text { ( } v \text {. combined conventional cohort) } \\
\text { Increased with risperidone ( } v \text {.haloperidol) } \\
\text { No increase with clozapine or olanzapine } \\
\text { ( } v \text {. haloperidol) } \\
\text { Decreased with quetiapine ( } v \text {. haloperidol) }\end{array}$ \\
\hline $\begin{array}{l}\text { Cunningham et al } \\
\text { (2003) }\end{array}$ & Veterans database & Independent & $\begin{array}{l}\text { Increased with risperidone and olanzapine } \\
\text { No increase with quetiapine }\end{array}$ \\
\hline
\end{tabular}

GP, general practitioner.

I. BMS, Bristol-Myers Squibb; Janssen, Janssen-Cilag; Lilly, Eli Lilly \& Co.

patients treated with atypical antipsychotics compared with those taking conventional agents (Table 3; Sernyak et al, 2002; Koro et al, 2002; Lambert et al, 2002; Cunningham et al, 2003). However, several studies contradict these findings. For example, Buse et al (2003) found no significant difference in the risk of developing diabetes between the combined cohorts of those taking atypical antipsychotics and those taking conventional drugs.
One of the best pharmaco-epidemiological studies from a methodological perspective is that by Cunningham et al (2003). This study has not yet been published in full, so its findings must be interpreted with caution. Nevertheless, the study had the advantage of being independent of pharmaceutical industry sponsorship, and the data are adjusted for several important diabetes risk factors, including age, race and the use of other diabetogenic drugs. The study 
found that olanzapine, quetiapine and risperidone were all associated with a similarly increased risk of diabetes compared with conventional antipsychotics, although in the case of quetiapine this did not reach statistical significance - possibly owing to the smaller sample size. With all three drugs, the hazard ratio was approximately 1.5 compared with conventional antipsychotics.

In the above study and in that by Sernyak et al (2002), the increased risk of diabetes in users of atypical $v$. conventional drugs was only apparent in younger age groups. This suggests that if atypical drugs are a causal factor in younger age groups then they may be bringing forward the presentation of an underlying diathesis rather than precipitating diabetes de novo.

Pharmaco-epidemiological studies can only show an association; they cannot prove causality. Thus, the higher rate of diabetes seen in antipsychotic users compared with non-users may represent a causal effect of the drugs, but other explanations must also be considered, including the possible effect of schizophrenia itself, the possibility that some patients receiving antipsychotic medications are more likely to have blood tests that detect diabetes than patients who are not taking these drugs, and the possibility that those using antipsychotics may have more risk factors for diabetes, such as poor diet, lack of exercise and obesity. Similarly, the higher rates of diabetes seen in patients receiving atypical rather than conventional antipsychotics may represent a true differential effect of the medications in their ability to cause diabetes, but alternatively may represent differential screening for diabetes and/or differential loading of risk factors among users of different classes of antipsychotic.

The question of whether individual atypical antipsychotic agents differ in their propensity to cause diabetes is important, but owing to their methodological weaknesses, pharmaco-epidemiological studies cannot provide a definitive answer. Most of the studies in Tables 2 and 3 did not have the power to compare the relative risk of diabetes between individual atypical drugs. Of those that did, the results are conflicting. For example, Fuller et al (2003) found that olanzapine was associated with an increased risk of developing diabetes compared with risperidone, whereas Buse et al (2003) found no significant difference between these two drugs.

\section{DISCUSSION}

Diabetes mellitus is a common and frequently undiagnosed condition for which there are many risk factors, possibly including schizophrenia itself. An association between antipsychotic drugs and diabetes was first suggested in the 1950s, but recent interest has focused primarily on the atypical antipsychotics. Much of the available data are non-prospective, and, owing to various methodological weaknesses, only limited conclusions can be drawn.

Cross-sectional studies show that the prevalence of diabetes and impaired glucose tolerance among patients with schizophrenia is higher than in comparative populations, and that much of this morbidity is undiagnosed. For several antipsychotic agents (e.g. chlorpromazine, clozapine, olanzapine, risperidone and quetiapine) a subset of anecdotal reports describe diabetes appearing soon after the patient starts taking the drug, resolving on stopping it, and in some cases reappearing with a further drug challenge. These cases indicate that antipsychotics can cause diabetes in some patients, but they cannot determine the incidence or whether the risk varies between different antipsychotics. Some retrospective case-note studies have shown a significant increase in blood glucose levels during treatment with several antipsychotic medications (e.g. olanzapine, haloperidol and clozapine), but one cannot assume that this represents a causal relationship.

Pharmaco-epidemiological studies have the advantage of assessing large cohorts of patients, but there is no standardisation in terms of detection and diagnosis of diabetes, and many risk factors are not controlled for. Fairly consistent findings across these studies are a higher rate of diabetes in patients receiving atypical antipsychotic medications compared with those not receiving antipsychotics or compared with those receiving conventional agents. Studies attempting to establish whether the association with diabetes varies between atypical antipsychotic medications are contradictory and inconclusive.

In summary, non-prospective studies suggest that both conventional and atypical antipsychotic medications can cause diabetes mellitus in some patients. Patients and clinicians need to be aware of this risk. However, retrospective studies cannot quantify this risk or determine whether it varies between drugs and how it compares with recognised risk factors such as race, increasing age and obesity. These are important clinical questions, but they can only be answered by well-designed prospective studies that take account of potential confounders.

\section{REFERENCES}

Adams, P. F. \& Marano, M. A. (1995) Current

estimates for the national health interview survey, 1994 National Center for Health Statistics, Vital Health Statistics, 10(193)

Bennett, N., Dodd, T., Flatley, J., et al (1995) Health Survey for England 1993. London: HMSO.

Braceland, F. J., Meduna, L. J., Vaichulis, J. A. (1946) Delayed action of insulin in schizophrenia. American Journal of Psychiatry, 102, 108-109.

Brown, S., Birtwistle, J., Roe, L., et al (1999) The unhealthy lifestyle of people with schizophrenia. Psychological Medicine, 29, 697-701.

Buse, J. B., Cavazzoni, P., Hornbuckle, K., et al (2003) A retrospective cohort study of diabetes mellitus and antipsychotic treatment in the United States. Journal of Clinical Epidemiology, 56, 164-170.

Cavazzoni, P., Hornbuckle, K., Wu, J., et al (2002) A retrospective cohort study of diabetes mellitus and antipsychotic treatment in the United Kingdom. International Journal of Neuropsychopharmacology, $\mathbf{5}$ (suppl. I), SI68.

Citrome, L. (2003) Antipsychotic medication treatment and new prescriptions for insulin and oral hypoglycaemics. European Neuropsychopharmacology, 13 (suppl. 4), S306,

Cooperberg, A. A. \& Eidlow, S. (1956) Haemolytic anaemia, jaundice and diabetes mellitus following chlorpromazine therapy. Journal of the Canadian Medical Association, 75, 746-749.

Cunningham, F., Lambert, B., Dalack, G., et al (2003) Diabetes associated with antipsychotic use in veterans with schizophrenia. Pharmacoepidemiology and Drug Safety, 12 (suppl. I), SI54-SI55.

Dixon, L., Weiden, P., Delahany, J., et al (2000) Prevalence and correlates of diabetes in national schizophrenia samples. Schizophrenia Bulletin, 26 903-912.

Freeman, H. (1946) Resistance to insulin in mentally disturbed soldiers. Archives of Neurology and Psychiatry, 56, 74-77.

Fuller, M. A., Shermock, K. M., Secic, M., et al (2003) Comparative study of the development of diabetes in patients taking risperidone and olanzapine. Pharmacotherapy, 23, 1037-1043.

Gianfrancesco, F. D., Grogg, A. L., Mahmoud, R. A., et al (2002) Differential effects of risperidone, olanzapine, clozapine, and conventional antipsychotics on type 2 diabetes: findings from a large health plan database. Journal of Clinical Psychiatry, 63, 920-930.

Hedenmalm, K., Hagg, S., Syahl, M., et al (2002) Glucose intolerance with atypical antipsychotics. Drug Safety, 25, II07-1II6.

Hiles, B. W. (1956) Hyperglycemia and glucosuria following chlorpromazine therapy (letter). JAMA, $\mathbf{1 6 2}$, 1651.

Koller, E. A. \& Doraiswamy, P. M. (2002) Olanzapineassociated diabetes mellitus. Pharmacotherapy, $\mathbf{2 2}$, $841-852$. 
Koller, E., Schneider, B., Bennett, K., et al (200I) Clozapine-associated diabetes. American Journal of Medicine, III, 716-723.

Koller, E. A., Cross, J.T., Doraiswamy, P. M., et al (2003) Risperidone-associated diabetes mellitus: a pharmacovigilance study. Pharmacotherapy, 23, 735-744.

Koller, E. A., Cross, J.T., Weber, J., et al (2004) Quetiapine-associated diabetes mellitus. Journal of Clinical Psychiatry, in press.

Koro, C. E., Fedder, D. O, L'Italien, G. J., et al (2002) Assessment of independent effect of olanzapine and risperidone on risk of diabetes among patients with schizophrenia: population based nested case control study. BMJ, 325, 243-248.

Lambert, B., Chou, C. H., Chang, K. Y., et al (2002) Assessing the risk of antipsychotic-induced type II diabetes among schizophrenics: a matched case-contro study. European Neuropsychopharmacology 12 (suppl. 3), S307.

Lorenz, W. (1922) Sugar tolerance in dementia praecox and other mental disorders. Archives of Neurology and Psychiatry, 8, 184-196.

Meyer, J. M. (2002) A retrospective comparison of weight, lipid, and glucose changes between risperidoneand olanzapine-treated inpatients: metabolic outcome after I year. Journal of Clinical Psychiatry, 63, 425-433.

Mukherjee, S., Schnur, D. B., Reddy, R. (1989) Family history of type 2 diabetes in schizophrenic patients. Lancet, i, 495.

Mukherjee, S., Decina, P., Bocola, V., et al (1996)

Diabetes mellitus in schizophrenic patients.

Comprehensive Psychiatry, 36, 68-73.

Ryan, M. C. M. \& Thakore, J. H. (2002) Physical consequences of schizophrenia and its treatment: the metabolic syndrome. Life Sciences, 7l, 239-257.

Ryan, M. C., Collins, P., \& Thakore, J. H. (2003)

Impaired fasting glucose tolerance in first-episode, drug naïve patients with schizophrenia. American Journal of Psychiatry, 160, 284-289.

Sernyak, M. J., Leslie, D. L., Alarcon, R. D., et at (2002) Association of diabetes mellitus with use of atypical neuroleptics in the treatment of schizophrenia. American Journal of Psychiatry, 159, 56I-566.

Subramaniam, M., Chong, S. A. \& Pek, E. (2003)

Diabetes mellitus and impaired glucose tolerance in patients with schizophrenia Canadian Journal of Psychiatry, 48, 345-347.

Tabata, H., Kikuoka, M., Kikuoka, H., et al (1987) Characteristics of diabetes mellitus in schizophrenic

\section{CLINICAL IMPLICATIONS}

Cross-sectional studies indicate that the prevalence of diabetes mellitus and impaired glucose tolerance is higher in patients with schizophrenia than in the general population, with much of this morbidity being undiagnosed.

- Non-prospective studies suggest that both conventional and atypical antipsychotic medications can cause diabetes in some individuals.

Atypical antipsychotic agents appear to increase the risk of diabetes more than conventional antipsychotics do, but differential loading of risk factors for diabetes between these two groups and/or more intensive screening with atypical agents may account for these differences.

\section{LIMITATIONS}

- Retrospective data are not available for several atypical antipsychotic medications, including ziprasidone, aripiprazole and zotepine.

- Retrospective studies can only establish an association between antipsychotic medications and diabetes; they cannot establish causality.

Well-designed prospective studies are needed to quantify the risk associated with antipsychotic medications, to determine whether the risk varies between agents, and how it compares with other recognised risk factors for diabetes.

PETER M. HADDAD, MD, MRCPsych, Cromwell House, Bolton, Salford and Trafford Mental Health NHS Trust, Cromwell Road, Eccles, Salford M30 0GT, UK

patients. Journal of the Medical Association of Thailand, 70 90-93.

Taylor, D., Young, C., Mahomed, R., et al (2003) A prevalence study of impaired glucose tolerance and diabetes mellitus in hospitalised patients with schizophrenia European Neuropsychopharmacology, 13 (suppl. 4), S323.

Thakore, J. H., Mann, J. N., Vlahos, I., et al (2002) ncreased visceral fat distribution in drug-naïve and drug-free patients with schizophrenia International Journal of Obesity, 26, 137-141.
Thonnard-Neumann, E. (1968) Phenothiazines and diabetes in hospitalized women. American Journal of Psychiatry, 124, 978-982.

Wirshing, D. A., Boyd, J. A., Meng, L. R., et al (2002) The effects of novel antipsychotics on glucose and lipid levels. Journal of Clinical Psychiatry, 63, 856-865.

Yu, B. P., Ortiz, T., Chong, Y. S., et al (2002) Atypica antipsychotics in patients with diabetes mellitus: a retrospective review. Behavioral Health Trends, 14, 42 45 